\title{
A New LMI-Based Robust Sliding Mode Control for the Uncertain Discrete-Time Systems
}

\author{
Ahmadreza Argha, Li Li, Steven W. Su^ and Hung Nguyen
}

\begin{abstract}
In this paper, a new approach for designing a robust Discrete-time Sliding Mode Control (DSMC) is proposed for the uncertain discrete-time systems. To this end, an LMI approach is used to develop a new framework to design the linear sliding functions which are linear to the state. The LMI approach proposed in this paper is designed to deal with uncertain systems (matched and unmatched). It is wellknown that the finite sampling rate for the discrete-time systems leads to this fact that state move within a bound around the predetermined sliding surface referred to as quasi-sliding mode band. In this paper, this matter will be discussed in a new point of view and an innovative method will be used to obtain the ultimate bound on the system state.
\end{abstract}

\section{INTRODUCTION}

Sliding mode control (SMC) is, basically, referred to as a nonlinear control strategy and, in addition, a special kind of Variable structure control (VSC) which changes its structure automatically in order to improve the dynamics performance. The basic idea of SMC is to drive the state trajectories into a predetermined sliding surface and, then, maintain on the sliding surface thereafter. SMC has been always reputed for its robustness, as this control strategy provides dynamics with an invariance property to the matched uncertainties and external disturbances [1]. Consequently, SMC can be used for the systems involved the modeling uncertainties and, especially, it is useful when the exact model of the dynamics is not available. However, there is a significant drawback for SMC known as chattering, due to its discontinuous control part. Softening the discontinuous control part is used as an approach. Hence, the discontinuous term is replaced by a continuous approximation for reducing the chattering phenomena [2].

Discrete-time sliding mode control (DSMC) was firstly considered in the mid 80s [4]. This idea was continued by a wide range of publications [5] - [10]. On the other hand, it is well-known that the finite sampling rate in the discrete-time systems would result in this fact that state trajectories could not stay on the ideal sliding surface. Instead, state trajectories would move within a band around the predetermined sliding surface referred to as quasi-sliding mode band [3].

Broadly speaking, the method of designing the sliding surface for DSMC is exactly the same as the one used for the continuous SMC (CSMC). For designing the sliding surface, a number of different approaches have been proposed by far;

Ahmadreza Argha, Li Li, Steven W. Su and Hung Nguyen are with Faculty of Engineering and Information Technology, University of Technology, Sydney, PO Box 123, Broadway, NSW 2007, Australia.

\{Ahmadreza.Argha, Li.Li, Steven.Su, Hung. Nguyen\}@uts . edu . au such as pole placement, eigenstructure assignment, optimal quadratic methods [2]. Besides, LMI methods have been explored in [11] - [13]. Moreover, the problem of robust design approach which aims to make the reduced order dynamics [2] insensitive to unmatched uncertainties has been studied in [2], [14]. However, the robustness of the system during the reaching phase is also of importance. This issue is more critical when there is a large initial distance from the predetermined switching surface which may lead to excessively large control inputs, especially for the controllers without reaching law.

A large number of the early DSMC publications have focused on creating a discrete-time counterpart to the continuous-time reachability condition [15] - [17]. Furthermore, for DSMC, elimination of the discontinuous control part from the control law leads to the eliminating the chattering issue [21], [20], [22]. The obtained control law is called linear control law or equivalent control law. Indeed, it is argued that DSMC does not necessarily require the use of a variable structure discontinuous control strategy [21], [20], [22]. References [21], [20] have shown that using the pure equivalent control can ensure that the state trajectories stay within a neighbourhood of the sliding surface in the presence of bounded matched uncertainty. Moreover, according to the results presented in [21], [20] the use of a switching function in the control law may not necessarily improve the performance. Note that, obviously, the DSMC problem using only equivalent control law can be regarded as a robust optimal control problem and it will be equivalent to discrete-time Lyapunov min-max problems [18] or discretetime Riccati min-max problems [19].

Mainly, the robust optimal control strategy used for designing DSMC has been considered for the systems with matched uncertainties and/or external disturbances. In this paper, our major focus is on the problem of designing DSMC for the discrete-time systems involving unmatched uncertainties and matched disturbances using an LMI approach. Indeed, here, in order to design a robust sliding surface, an innovative LMI method will be developed. To achieve this goal, the sliding surface is designed in a way (using LMI approach) so that the closed-loop system during the socalled quasi sliding mode is insensitive against unmatched uncertainties. Besides, a new framework will be used to find the ultimate bound on the underlying system state. Furthermore, the proposed method of this paper can be applied to the systems whose open-loop dynamics are not necessarily stable.

The rest of this paper is organized as follows: Section 
II describes the problem formulation. In Section III, the proposed method of this paper to design the sliding surface is given. Section IV explains the use of practical DSMC for the systems including uncertainties and disturbances. Effectiveness of the proposed DSMC is studied by numerical examples in Section V. Finally, Section VI concludes this paper.

\section{Problem Formulation}

Consider the following uncertain linear discrete-time system,

$$
x(k+1)=[A+\Delta A(k)] x(k)+B[u(k)+f(k)],
$$

where $x(k) \in R^{n}$ and $u(k) \in R^{m}$. Without loss of generality, it is assumed that $B \in R^{n \times m}$ and $m \leq n$. Besides, $\operatorname{rank}(B)=m$ and it is assumed that the pair $(A, B)$ is stabilizable. The uncertain matrix $\Delta A(k)=M R(k) N$, where matrices $M$ and $N$ are known and $R(k)$ is an unknown matrix satisfying $R^{T}(k) R(k) \leq I, \forall k \geq 0 ; f(k)$ denotes external disturbance with known bound.

The following lemmas are useful in the sequel.

Lemma 1 ( [24]): Let $E, F(k)$ and $G$ be real matrices of appropriate dimensions with $F^{T}(k) F(k) \leq I, \forall k \geq 0$, then, for any scalar $\varepsilon>0$, we have

$$
E F(k) G+G^{T} F^{T}(k) E^{T} \leq \varepsilon E E^{T}+\varepsilon^{-1} G^{T} G .
$$

Lemma 2 (Schur Complement [23]): The following linear matrix inequality,

$$
\left[\begin{array}{cc}
Q_{1} & H \\
H^{T} & Q_{2}
\end{array}\right]>0
$$

is equivalent to $Q_{2}>0, Q_{1}-H Q_{2}^{-1} H^{T}>0$, where $Q_{1}^{T}=Q_{1}$, $Q_{2}^{T}=Q_{2}$ and $H$ is a matrix with appropriate dimension.

\section{Designing Discrete-time SMC}

Consider the following linear discrete-time sliding function:

$$
\sigma_{x}(k)=S x(k),
$$

where $S \in \mathbb{R}^{m \times n}$ will be designed later such that $S B$ is nonsingular. During the ideal sliding motion the sliding function satisfies:

$$
\sigma_{x}(k+1)=\sigma_{x}(k)=0, \quad \forall k>k_{s},
$$

where $k_{s}>0$ denotes the time that sliding motion starts. Hence, the so-called equivalent control law may be obtained from (1), (2) and (3) as

$$
u_{e q}(k)=-(S B)^{-1} S[A+\Delta A(k)] x(k)-f(k) .
$$

Substituting the control law in (4) in the system (1), one may achieve the following dynamics which presents the ideal sliding mode dynamics,

$$
x(k+1)=[A+\Delta A(k)-\Delta \hat{A}] x(k),
$$

where $\Delta \hat{A}=B(S B)^{-1} S[A+\Delta A(k)]$.
Theorem 1: The uncertain sliding dynamics in (5) is asymptotically stable, if there exist a symmetric matrix $\bar{P}>0$, a matrix $X$ and a scalar $\varepsilon>0$ satisfying the following LMI:

$$
\left[\begin{array}{ccc}
-\bar{P} & \bar{P} A^{T}+X^{T} B^{T} & \bar{P} N^{T} \\
A \bar{P}+B X & -\bar{P}+\varepsilon M M^{T} & 0 \\
N \bar{P} & 0 & -\varepsilon I
\end{array}\right]<0,
$$

where $S=B^{T} \bar{P}^{-1}$.

Proof: We choose the following Lyapunov function candidate:

$$
V(x(k))=x^{T}(k) P x(k) .
$$

Now, it follows from (5) that

$$
\begin{aligned}
\Delta V(x(k))= & V(x(k+1))-V(x(k)) \\
= & x^{T}(k)\left[A+\Delta A(k)-\hat{A}_{\Delta}\right]^{T} P\left[A+\Delta A(k)-\hat{A}_{\Delta}\right] x(k) \\
& -x^{T}(k) P x(k) .
\end{aligned}
$$

With the choice of $S=B^{T} P$, one may derive that

$$
\begin{aligned}
\Xi:= & {\left[A+\Delta A(k)-\hat{A}_{\Delta}\right]^{T} P\left[A+\Delta A(k)-\hat{A}_{\Delta}\right]-P } \\
= & (A+\Delta A(k))^{T} P(A+\Delta A(k)) \\
& -(A+\Delta A(k))^{T} P B\left(B^{T} P B\right)^{-1} B^{T} P(A+\Delta A(k))-P .
\end{aligned}
$$

It is known that the feasibility of $\Xi<0$ is equivalent to the feasibility of the following term.

$$
\begin{aligned}
(A & +\Delta A(k))^{T} P(A+\Delta A(k))+\left[(A+\Delta A(k))^{T} P B\left(B^{T} P B\right)^{-1}\right. \\
& \left.+F^{T}\right]\left(B^{T} P B\right)\left[F+\left(B^{T} P B\right)^{-1} B^{T} P(A+\Delta A(k))\right] \\
& -(A+\Delta A(k))^{T} P B\left(B^{T} P B\right)^{-1} B^{T} P(A+\Delta A(k))-P<0,
\end{aligned}
$$

where $F$ is an introduced auxiliary variable. Equivalently,

$$
\begin{aligned}
(A+\Delta A(k))^{T} P(A+\Delta A(k))+F^{T} B^{T} P B F+(A+\Delta A(k))^{T} \\
\quad \times P B F+F^{T} B^{T} P(A+\Delta A(k))-P<0,
\end{aligned}
$$

or

$\Sigma_{1}:=\left[(A+\Delta A(k))^{T}+F^{T} B^{T}\right] P[(A+\Delta A(k))+B F]-P<0$.

Let $\bar{P}=P^{-1}$, then, $\Sigma_{1}$ is equivalent to:

$$
\begin{aligned}
\Sigma_{2}:=\bar{P}[(A+\Delta A(k))+B F]^{T} \bar{P}^{-1}[(A+\Delta A(k)) & +B F] \bar{P} \\
& -\bar{P}<0 .
\end{aligned}
$$

According to Lemma $2, \Sigma_{2}$ is equivalent to:

$$
\Sigma_{3}:=\left[\begin{array}{cc}
-\bar{P} & \bar{P}[(A+\Delta A(k))+B F]^{T} \\
{[(A+\Delta A(k))+B F] \bar{P}} & -\bar{P}
\end{array}\right]<0 .
$$

Introducing $X=F \bar{P}$, we have

$$
\begin{aligned}
\Sigma_{3}:= & {\left[\begin{array}{cc}
-\bar{P} & \bar{P} A^{T}+X^{T} B^{T} \\
A \bar{P}+B X & -\bar{P}
\end{array}\right] } \\
& +\left[\begin{array}{cc}
0 & \bar{P} \Delta A^{T}(k) \\
\Delta A(k) \bar{P} & 0
\end{array}\right]<0 .
\end{aligned}
$$


Now, the second matrix in the above equation can be rearranged as

$$
\begin{aligned}
& {\left[\begin{array}{cc}
0 & \bar{P} \Delta A^{T}(k) \\
\Delta A(k) \bar{P} & 0
\end{array}\right]=\left[\begin{array}{cc}
0 & \bar{P} N^{T} R^{T}(k) M^{T} \\
M R(k) N \bar{P} & 0
\end{array}\right] } \\
= & {\left[\begin{array}{c}
\bar{P} N^{T} \\
0
\end{array}\right] R^{T}(k)\left[\begin{array}{ll}
0 & M^{T}
\end{array}\right]+\left[\begin{array}{c}
0 \\
M
\end{array}\right] R(k)\left[\begin{array}{ll}
N \bar{P} & 0
\end{array}\right] } \\
\leq & \varepsilon\left[\begin{array}{c}
0 \\
M
\end{array}\right]\left[\begin{array}{ll}
0 & M^{T}
\end{array}\right]+\varepsilon^{-1}\left[\begin{array}{c}
\bar{P} N^{T} \\
0
\end{array}\right]\left[\begin{array}{ll}
N \bar{P} & 0
\end{array}\right],
\end{aligned}
$$

where $\varepsilon>0$ is a scalar. Note that Lemma 1 is used to obtain the above inequality. From Equations (12), (13) and Lemma 2, it can be shown that $\Sigma_{3}<0$ is implied by the LMI (6). In the case that LMI (6) is feasible, one can obtain $S=B^{T} P=B^{T} \bar{P}^{-1}$.

Remark 1: It should be mentioned that the equivalent control law in (4) is not a practical controller, since it has some unknown uncertain terms. The equivalent controller has been obtained in order to find the sliding mode dynamics (5). To induce and maintain a sliding motion, a control law is designed in the sequel.

Remark 2: Note that the equation in (9) has the form of algebraic Riccati equation which is not possible to rewrite it as an LMI in the current form. Hence, we have gradually transformed it to a special Lyapunov equation. Furthermore, the control law in (4) is referred to as Lyapunov minmax controller, since it minimizes the Lyapunov function's difference, $\Delta V$, for the worst case of the uncertainty; see e.g. [25].

Remark 3: It is obvious that the stabilizability of the matrix pair $(A, B)$ is the necessary condition for the feasibility of the inequalities (10) and (11), but not the sufficient condition.

Note that in [26] another framework is used to deal with the uncertain inequality in (9) and convert it to an LMI. Here, our proposed method removed the stability assumption in [26] and also improved the conservatism of the control method in [26].

Consider the following inequality which is used in [26] to derive the LMI condition,

$$
\begin{aligned}
& {\left[A+\Delta A(k)-\hat{A}_{\Delta}\right]^{T} P\left[A+\Delta A(k)-\hat{A}_{\Delta}\right]} \\
& \quad \leq 2(A+\Delta A(k))^{T} P(A+\Delta A(k)) \\
& \quad+2(A+\Delta A(k))^{T} S^{T}(S B)^{-1} S(A+\Delta A(k)) .
\end{aligned}
$$

By substituting the inequality (14) in (8), one may found that

$$
\Delta V(x(k)) \leq x^{T}(k) \Pi x(k),
$$

where $\Pi=2(A+\Delta A(k))^{T} P(A+\Delta A(k))+2(A+$ $\Delta A(k))^{T} S^{T}(S B)^{-1} S(A+\Delta A(k))$. Using Lemmas 1 and 2 , it can be shown that $\Pi<0$ is implied by the following LMI:

$$
\left[\begin{array}{cccc}
-P+\varepsilon N^{T} N & \sqrt{2} A^{T} P & \sqrt{2} A^{T} P B & 0 \\
\sqrt{2} P A & -P & 0 & \sqrt{2} P M \\
\sqrt{2} B^{T} P A & 0 & -B^{T} P B & \sqrt{2} B^{T} P M \\
0 & \sqrt{2} M^{T} P & \sqrt{2} M^{T} P B & -\varepsilon I
\end{array}\right]<0
$$

where $P>0$ is a symmetric matrix and $\varepsilon>0$ is a scalar. Obviously, regardless of the fact that the dimension of this LMI is bigger than the dimension of LMIs given in Theorem 1, the necessary condition for the feasibility of LMI (15) is the stability of $\sqrt{2} A$. To address this strong assumption arising from the inequality (14), we suggest to use a fictitious state-feedback as:

$$
\begin{aligned}
& {\left[A+B K+\Delta A(k)-B(S B)^{-1} S(A+B K+\Delta A(k))\right]^{T} P} \\
& \quad \times\left[A+B K+\Delta A(k)-B(S B)^{-1} S(A+B K+\Delta A(k))\right] \\
& \quad \leq 2(A+B K+\Delta A(k))^{T} P(A+B K+\Delta A(k)) \\
& \quad+2(A+B K+\Delta A(k))^{T} S^{T}(S B)^{-1} S(A+B K+\Delta A(k)),
\end{aligned}
$$

where $K$ is a known matrix which can stabilize the pair $(A, B)$. Eventually, LMI (15) will be changed to the LMI in (17), shown at the top of the next page, where, again, $P>0$ is a symmetric matrix and $\varepsilon>0$ is a scalar. Clearly, the LMI (17) is less conservative compared to the one in (15). This idea will be used in the next section to design DSMC controller.

\section{SLIding Mode Controller}

One may obtain from (1) and (2) that

$$
\sigma_{x}(k+1)=S[A+\Delta A] x(k)+S B[u(k)+f(k)] .
$$

Assume

$$
f_{i}^{l} \leq f_{i} \leq f_{i}^{u}, \quad i=1, \cdots, m
$$

Define,

$$
f_{i}^{+}=\frac{f_{i}^{u}+f_{i}^{l}}{2}, f_{i}^{-}=\frac{f_{i}^{u}-f_{i}^{l}}{2}, \quad i=1, \cdots, m,
$$

and

$$
\begin{aligned}
\mathscr{D}^{+} & =\operatorname{col}\left(f_{1}^{+}, \cdots, f_{m}^{+}\right), \mathscr{D}^{-}=\operatorname{diag}\left(f_{1}^{-}, \cdots, f_{m}^{-}\right) \\
\mathscr{D}_{c} & =\operatorname{col}\left(f_{1}^{-}, \cdots, f_{m}^{-}\right) .
\end{aligned}
$$

Now, the following control law is proposed,

$$
u(k)=-(S B)^{-1} S A x(k)-\mathscr{D}^{+}-\mathscr{D}^{-} \operatorname{sgn}\left(\sigma_{x}(k)\right) .
$$

Remark 4: Note that, here, to construct the control law (22), we just used the known upper and lower bounds on matched disturbance. Although it can be seen in the literature that the term $\triangle A x(k)$ or $S \Delta A x(k)$ is assumed to be bounded; see e.g. [26], [27] and [28], this assumption implies the boundedness of system states, thus, the stability of the system from the first. Hence, in this paper, we construct the nonlinear part of the controller by using only the external disturbance information.

The sequel of this paper, aims to consider the stability of the system (1) using the controller (22). Hence, applying the controller (22) to the system (1), it is seen that

$$
\begin{aligned}
x(k+1)=(A & +\Delta A-\hat{A}) x(k) \\
& +B\left[f(k)-\mathscr{D}^{+}-\mathscr{D}^{-} \operatorname{sgn}\left(\sigma_{x}(k)\right)\right],
\end{aligned}
$$

where $\hat{A}=B(S B)^{-1} S A$. Note that, following the idea used in (16), again, equation (23) can be revised to:

$$
\begin{gathered}
x(k+1)=\left[A+B K+\Delta A-B(S B)^{-1} S(A+B K)\right] x(k) \\
+B\left[f(k)-\mathscr{D}^{+}-\mathscr{D}^{-} \operatorname{sgn}\left(\sigma_{x}(k)\right)\right],
\end{gathered}
$$




$$
\left[\begin{array}{cccc}
-P+\varepsilon N^{T} N & \sqrt{2}(A+B K)^{T} P & \sqrt{2}(A+B K)^{T} P B & 0 \\
\sqrt{2} P(A+B K) & -P & 0 & \sqrt{2} P M \\
\sqrt{2} B^{T} P(A+B K) & 0 & -B^{T} P B & \sqrt{2} B^{T} P M \\
0 & \sqrt{2} M^{T} P & \sqrt{2} M^{T} P B & -\varepsilon I
\end{array}\right]<0
$$

in which matrix $K$ is a stabilizing state-feedback such that $A+B K$ is stable. Furthermore, it can be found that

$$
\sigma_{x}(k+1)=S \Delta A x(k)+S B\left[f(k)-\mathscr{D}^{+}-\mathscr{D}^{-} \operatorname{sgn}\left(\sigma_{x}(k)\right)\right],
$$

The following lemma is given to characterize the boundedness of the state of the system (23).

Lemma 3 ( [23]): Let $V(\zeta(k))$ be a Lyapunov candidate function. In the case that there exist real scalars $v \geq 0, \alpha>0$, $\beta>0$ and $0<\rho<1$ such that

$$
\alpha\|\zeta(k)\|^{2} \leq V(\zeta(k)) \leq \beta\|\zeta(k)\|^{2}
$$

and

$$
V(\zeta(k+1))-V(\zeta(k)) \leq v-\rho V(\zeta(k)),
$$

then $\zeta(k)$ will satisfy

$$
\|\zeta(k)\|^{2} \leq \frac{\beta}{\alpha}\|\zeta(0)\|^{2}(1-\rho)^{k}+\frac{v}{\alpha \rho} .
$$

Then every autonomous LTI discrete-time system satisfying the above lemma is said to be ultimately bounded.

Theorem 2: The control law (22) can drive the system state into a boundary layer around the ideal sliding surface (2) and, in addition, the system state is ultimately bounded if there exist a symmetric matrix $P>0$ and scalars $\varepsilon>0$ and $\eta>0$ satisfying the following LMI,

$$
\left[\begin{array}{cccc}
-P+\eta I+\varepsilon N^{T} N & (A+B K)^{T} P & 0 & 0 \\
P(A+B K) & -P & 0 & P M \\
0 & 0 & -B^{T} P B & 2 B^{T} P M \\
0 & M^{T} P & 2 M^{T} P B & -\varepsilon I
\end{array}\right]<0,
$$

in which the matrix $K$ is a known stabilizing state-feedback and $S=B^{T} P$.

Proof: Define

$$
V(\zeta(k))=x^{T}(k) P x(k)+\sigma_{x}^{T}(k)(S B)^{-1} \sigma_{x}(k),
$$

where $\zeta(k)=\left[\begin{array}{ll}x^{T}(k) & \sigma_{x}^{T}(k)\end{array}\right]^{T}$. Thus, we can write

$$
\begin{aligned}
\Delta V(\zeta(k))= & V(\zeta(k+1))-V(\zeta(k)) \\
= & x^{T}(k+1) \operatorname{Px}(k+1)+\sigma_{x}^{T}(k+1)(S B)^{-1} \sigma_{x}(k+1) \\
& -x^{T}(k) \operatorname{Px}(k)-\sigma_{x}^{T}(k)(S B)^{-1} \sigma_{x}(k) .
\end{aligned}
$$

Hence, from (24) and (25), it can be shown that

$$
\begin{aligned}
& \Delta V(\zeta(k)) \\
= & x^{T}(k)\left[A+B K+\Delta A-B(S B)^{-1} S(A+B K)\right]^{T} P \\
& \times\left[A+B K+\Delta A-B(S B)^{-1} S(A+B K)\right] x(k) \\
& +2 x^{T}(k)\left[A+B K+\Delta A-B(S B)^{-1} S(A+B K)\right]^{T} P \\
& \times B\left[f(k)-\mathscr{D}^{+}-\mathscr{D}^{-} \operatorname{sgn}\left(\sigma_{x}(k)\right)\right] \\
& +\left[f(k)-\mathscr{D}^{+}-\mathscr{D}^{-} \operatorname{sgn}\left(\sigma_{x}(k)\right)\right]^{T}\left(B^{T} P B\right) \\
& \times\left[f(k)-\mathscr{D}^{+}-\mathscr{D}^{-} \operatorname{sgn}\left(\sigma_{x}(k)\right)\right] \\
& +x^{T}(k) \Delta A^{T} S^{T}(S B)^{-1} S \Delta A x(k)+2 x^{T}(k) \Delta A^{T} S^{T} \\
& \times\left[f(k)-\mathscr{D}^{+}-\mathscr{D}^{-} \operatorname{sgn}\left(\sigma_{x}(k)\right)\right] \\
& +\left[f(k)-\mathscr{D}^{+}-\mathscr{D}^{-} \operatorname{sgn}\left(\sigma_{x}(k)\right)\right]^{T}\left(B^{T} P B\right) \\
& \times\left[f(k)-\mathscr{D}^{+}-\mathscr{D}^{-} \operatorname{sgn}\left(\sigma_{x}(k)\right)\right] \\
& -x^{T}(k) P x(k)-\sigma_{x}^{T}(k)(S B)^{-1} \sigma_{x}(k) .
\end{aligned}
$$

Besides, note that

$$
\begin{aligned}
& 2 x^{T}(k)\left[A+B K+\Delta A-B(S B)^{-1} S(A+B K)\right]^{T} P \\
& \times B\left[f(k)-\mathscr{D}^{+}-\mathscr{D}^{-} \operatorname{sgn}\left(\sigma_{x}(k)\right)\right] \\
&= 2 x^{T}(k) \Delta A^{T} S^{T}\left[f(k)-\mathscr{D}^{+}-\mathscr{D}^{-} \operatorname{sgn}\left(\sigma_{x}(k)\right)\right], \\
& x^{T}(k)\left[A+B K+\Delta A-B(S B)^{-1} S(A+B K)\right]^{T} P \\
& \times \\
&=x^{T}(k)\left[A+B K+\Delta A-B(S B)^{-1} S(A+B K)\right] x(k) \\
&-x^{T}(k)[A+B K]^{T} P B\left(B^{T} P B\right)^{-1} B^{T} P[A+B K] x(k) \\
&- 2 x^{T}(k) \Delta A^{T} P B\left(B^{T} P B\right)^{-1} B^{T} P[A+B K] x(k), \\
&- 2 x^{T}(k) \Delta A^{T} P B\left(B^{T} P B\right)^{-1} B^{T} P[A+B K] x(k) \\
& \leq x^{T}(k)[A+B K]^{T} P B\left(B^{T} P B\right)^{-1} B^{T} P[A+B K] x(k) \\
&+x^{T}(k) \Delta A^{T} P B\left(B^{T} P B\right)^{-1} B^{T} P \Delta A x(k), \\
& 2 x^{T}(k) \Delta A^{T} S^{T}\left[f(k)-\mathscr{D}^{+}-\mathscr{D}^{-} \operatorname{sgn}\left(\sigma_{x}(k)\right)\right] \\
& \leq x^{T}(k) \Delta A^{T} P B\left(B^{T} P B\right)^{-1} B^{T} P \Delta A x(k) \\
&+\left[f(k)-\mathscr{D}^{+}-\mathscr{D}^{-} \operatorname{sgn}\left(\sigma_{x}(k)\right)\right]^{T}\left(B^{T} P B\right) \\
& \times\left[f(k)-\mathscr{D}^{+}-\mathscr{D}^{-} \operatorname{sgn}\left(\sigma_{x}(k)\right)\right] .
\end{aligned}
$$

Also,

$$
\left\|f(k)-\mathscr{D}^{+}-\mathscr{D}^{-} \operatorname{sgn}\left(\sigma_{x}(k)\right)\right\| \leq 2\left\|\mathscr{D}_{c}\right\| .
$$

Applying (30)-(34) to (29), one may obtain that

$$
\begin{aligned}
\Delta V(\zeta(k)) & =V(\zeta(k+1))-V(\zeta(k)) \\
& \leq x^{T}(k) \Theta x(k)-\sigma_{x}^{T}(k)(S B)^{-1} \sigma_{x}(k)+\gamma,
\end{aligned}
$$

where $\Theta=\left\{[A+B K+\Delta A]^{T} P[A+B K+\Delta A]+\right.$ $\left.4 \triangle A^{T} P B\left(B^{T} P B\right)^{-1} B^{T} P \Delta A-P\right\}, \quad \gamma=16\|S B\|\left\|\mathscr{D}_{c}\right\|^{2}$. 
Using Lemmas 1,2 , it can easily be shown that $\Theta<-\eta I$ can be implied by the LMI (26). Besides, it is known that

$$
\begin{aligned}
\lambda_{\min }\left(\operatorname{diag}\left(P,(S B)^{-1}\right)\right) & \|\zeta(k)\|^{2} \leq V(\zeta(k)) \\
& \leq \lambda_{\max }\left(\operatorname{diag}\left(P,(S B)^{-1}\right)\right)\|\zeta(k)\|^{2}
\end{aligned}
$$

where $\lambda_{\min }(\cdot)$ and $\lambda_{\max }(\cdot)$ denote the minimum and maximum eigenvalues, respectively. Now, we know that

$$
\begin{aligned}
\Delta V(\zeta(k)) & =V(\zeta(k+1))-V(\zeta(k)) \\
& \leq-\eta x^{T}(k) x(k)-\sigma_{x}^{T}(k)(S B)^{-1} \sigma_{x}(k)+\gamma \\
& \leq-\frac{\min \left(\eta, \lambda_{\min }\left((S B)^{-1}\right)\right)}{\lambda_{\max }\left(\operatorname{diag}\left(P,(S B)^{-1}\right)\right)} V(\zeta(k))+\gamma,
\end{aligned}
$$

Thus, from Lemma 3, (37) and (36), it can be concluded that

$$
\begin{aligned}
& \forall \varepsilon_{1}>0, \exists k^{\star}>0, \text { s.t. } \forall k>k^{\star}, \\
& \|\zeta(k)\|^{2} \leq \frac{\lambda_{\max }\left(\operatorname{diag}\left(P,(S B)^{-1}\right)\right.}{\lambda_{\min }\left(\operatorname{diag}\left(P,(S B)^{-1}\right)\right) \cdot \min \left(\eta, \lambda_{\min }\left((S B)^{-1}\right)\right)} \gamma+\varepsilon_{1},
\end{aligned}
$$

where $\varepsilon_{1}>0$ is a scalar. Equation (38) implies that the system state is ultimately bounded and, in addition, during the sliding motion the state is within a bound around the sliding surface.

\section{A. Other sliding mode controllers}

1) Linear controller: Consider the following so-called linear controller,

$$
u(k)=-(S B)^{-1} S A x(k) .
$$

This linear controller can be used in the system (1) with external disturbance, and can guarantee the boundedness of the system state; in addition, in the absence of external disturbance the controller (39) can ensure the asymptotic stability of system (1). In this case, the ultimate bound on the closed-loop system state is as in (38) with $\gamma=4\|S B\|\|f\|^{2}$.

2) Using mean value of the disturbance in the controller: As discussed in section II, it is argued in the literature that the discontinuous part of the sliding mode control input can be detrimental to performance [6]. This issue is only true for the discrete-time systems involving balanced matched uncertainties and/or disturbances; see the Appendix A of [6]. However, for the unbalanced disturbances, the component $\mathscr{D}^{+}$can always be used to compensate the nonzero mean of the unbalanced matched disturbances. For instance, the controller in (39) can be revised to the following one; see [21],

$$
u(k)=-(S B)^{-1} S A x(k)-\mathscr{D}^{+} .
$$

3) Using switching components: The switching component in the discrete-time sliding mode controller would, basically, improve the performance for the cases that external disturbance is smooth enough. In simpler terms, using the switching functions in the discrete-time sliding mode control is more effective for the systems involving disturbance of which the maximum frequency component is small enough compared to the sampling rate of the discrete-time system. Note that under the smoothness and, in addition, boundedness conditions of external disturbance a disturbance
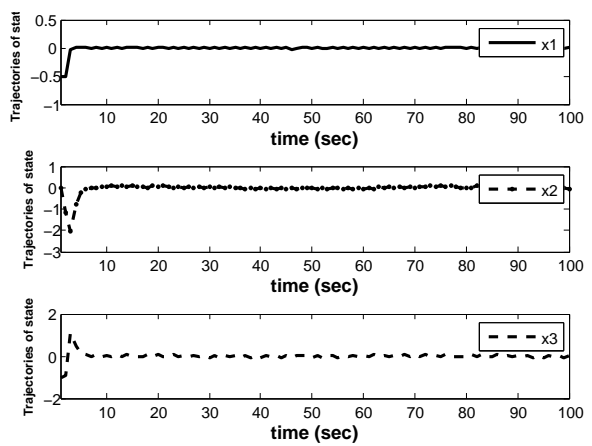

Fig. 1. Trajectories of the state for the nonlinear controller case

estimator has been proposed in [30] in order to reduce the ultimate bound on the discrete-time system state.

\section{Simulation Results}

Let parameters of system (1) to be as:

$$
\begin{aligned}
A & =\left[\begin{array}{ccc}
0.9 & 0.6 & 0.04 \\
-0.01 & 0.9 & 1.12 \\
-0.16 & -1.25 & 0.97
\end{array}\right], B=\left[\begin{array}{cc}
0.5 & 0 \\
0.09 & 0.1 \\
0.99 & 1.5
\end{array}\right] \\
M & =\left[\begin{array}{lll}
0.3 & 0.3 & 0.4
\end{array}\right]^{T}, N=\left[\begin{array}{lll}
-0.3 & 0.3 & 0.3
\end{array}\right], \\
R(k) & =0.6 \sin (k), f(k)=\left[\begin{array}{l}
0.02 \\
0.01
\end{array}\right] \sin \left(\frac{k}{10}\right) .
\end{aligned}
$$

The open-loop system is unstable, and the following statefeedback can stabilize the matrix pair $(A, B)$,

$$
K=\left[\begin{array}{ccc}
-1.2369 & -1.1773 & -0.6318 \\
0.9232 & 1.2143 & -0.6354
\end{array}\right]
$$

Using this state-feedback, the LMI (26) is solved and the results are as:

$$
\begin{aligned}
& P=\left[\begin{array}{ccc}
1.9518 & -0.3541 & -0.6148 \\
-0.3541 & 0.9710 & 0.7624 \\
-0.6148 & 0.7624 & 1.3873
\end{array}\right], \\
& \eta=0.1468 I_{3}, \varepsilon=2.9951 .
\end{aligned}
$$

Hence, using $P$, and also $\mathscr{D}^{+}=\left[\begin{array}{ll}0 & 0\end{array}\right]^{T}$ and $\mathscr{D}^{-}=$ $\left[\begin{array}{ll}0.02 & 0.01\end{array}\right]^{T}$ the control law in (22) is obtained. The results of applying this controller to the system (1) are shown in Figs. 1-2. Here, the initial state is assumed to be $x(0)=$ $\left[\begin{array}{lll}-0.5 & 0 & -1\end{array}\right]^{T}$. It can be seen that the state is bounded and also during the sliding motion the state trajectories are within a band of sliding surface $\sigma_{x}(k)=0$.

Once again, we repeat the above example by using linear controller only. Results are shown in Figs. 3-4. As seen, the performance of the linear controller is better.

\section{CONClusions}

In this paper, a new LMI based robust DSMC for the systems involving unmatched uncertainty and matched disturbance has been developed. Likewise, the proposed LMI method can deal with unstable systems. A new framework has also been used to find the ultimate bound on the system state. Furthermore, some notes on the using of the discontinuous term in the discrete-time sliding mode controller have been discussed. 


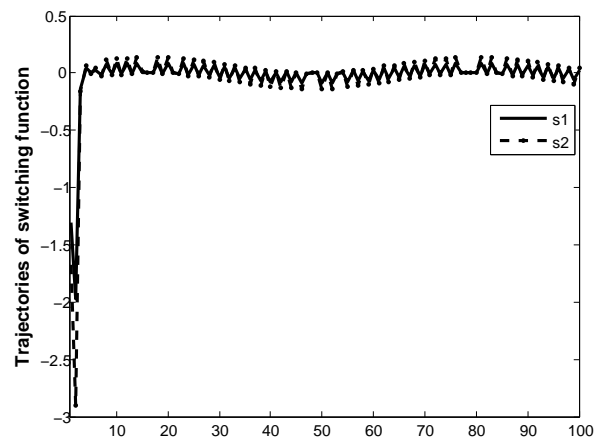

Fig. 2. Trajectories of the switching function for the nonlinear controller case
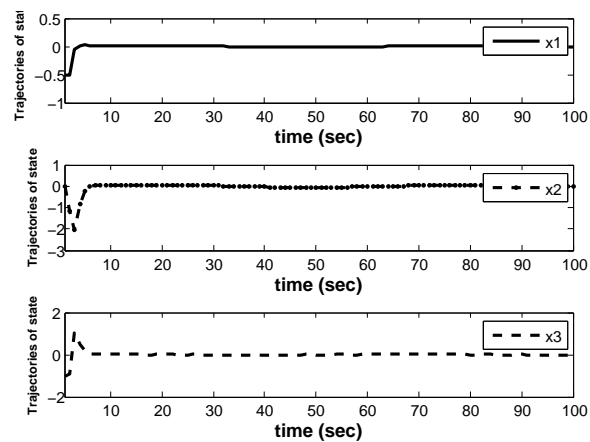

Fig. 3. Trajectories of the state for the linear controller case

\section{REFERENCES}

[1] V. I. Utkin, Variable structure systems with sliding modes, IEEE Trans. Automat. Contr., vol. AC-22, 1977, pp. 212-222.

[2] C. Edwards, S. K. Spurgeon, Sliding Mode Control: Theory and Applications, Taylor and Francis, London, 1998.

[3] W. Gao, Y. Wang and A. Homaifa, Discrete-time variable structure control system, IEEE Trans. Ind. Electron, vol. 42, 1995, pp 117-122.

[4] H. N. Iordanou and B. W. Surgenor, Experimental evaluation of the robustness of discrete sliding mode control versus linear quadratic control, IEEE Transactions on Control Systems Technology, vol. 5(2), 1997, pp. 254-260.

[5] A. Bartoszewicz, Discrete-time quasi-sliding-mode control strategies, IEEE Transactions on Industrial Electronics, vol. 45, 1998, pp. 633637.

[6] N. O. Lai, C. Edwards and S. K. Spurgeon, Discrete output feedback sliding-mode control with integral action, Int. J. Robust Nonlinear Control, vol. 16, 2006, pp. 21-43.

[7] V. Utkin, Sliding Modes in Control Optimization, Communications and Control Engineering Series, Springer-Verlag, London, 1992.

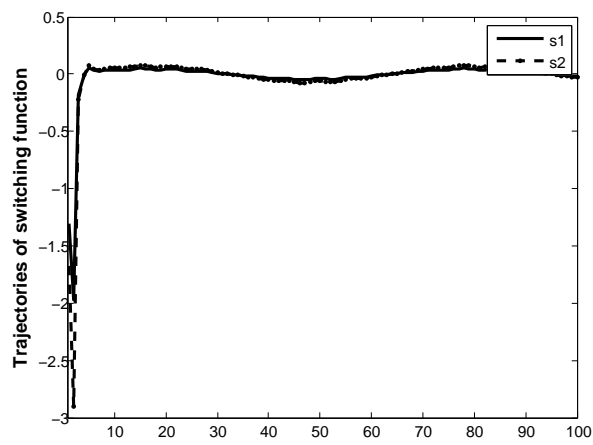

Fig. 4. Trajectories of the switching function for the linear controller case
[8] S. Qu, X. Xia, and J. Zhang, Dynamics of Discrete-time Sliding Mode Control Uncertain System with a Disturbance Compensator, IEEE Transactions on Industrial Electronics, vol. 61, no. 7, pp. 3502-3510, 2014.

[9] Q. Xu, Enhanced discrete-time sliding mode strategy with application to piezoelectric actuator control, IET Control Theory and Applications, vol. 7, Iss. 18, pp. 2153-2163, 2013.

[10] T. Bernardes, V. F. Montagner, H. A. Gründling, and H. Pinheiro, Discrete-Time Sliding Mode Observer for Sensorless Vector Control of Permanent Magnet Synchronous Machine, IEEE Transactions on Industrial Electronics, vol. 61, no. 4,pp. 1679-1691, 2014.

[11] C. Edwards, A practical method for the design of sliding mode controllers using linear matrix inequalities, Automatica, vol. 40, 2004 pp 1761-1769.

[12] G. Herrmann , S. K. Spurgeon, C. Edwards, A robust slidingmode output tracking control for a class of relative degree zero and nonminimum phase plants: A chemical process, International Journal of Control vol. 72, 2001, pp. 1194-1209.

[13] H. Choi, A new method for variable structure control system design: A Linear Matrix Inequality approach. Automatica, vol. 33, 1997, pp. 20892092. application

[14] K. Yasuda and Y. Nakatsuji, Robust Sliding Mode Control of Uncertain Systems, IEEE Workshop on Variable Structure Systems, Tokyo, Japan, 1996.

[15] A. J. Koshkouei and A. S. I. Zinober, Sliding mode control of discretetime systems, Journal of Dynamic Systems, Measurement, and Control, vol. 122, pp. 793-802, 2000.

[16] S.Z. Sapturk, Y. Istefanopulous, and O. Kaynak, On the stability of discrete-time sliding mode control systems, IEEE Transactions on Automatic Control, vol. 32, pp. 930-932, 1987.

[17] W. Gao, Y. Wang, and A. Homaifa, Discrete-time variable structure control systems, IEEE Transactions on Industrial Electronics, vol. 42, pp. 117-122, 1995.

[18] J. Manela, Deterministic control of uncertain linear discrete and sampled-data systems, Ph.D. Thesis, University of California, Berkeley, 1985.

[19] M. Corless, Stabilization of uncertain discrete-time systems, Proceedings of the IFAC Workshop on Model Error Concepts and Compensation, Boston, 1985.

[20] S. K. Spurgeon, Hyperplane design techniques for discrete-time variable structure control systems, International Journal of Control vol 55(2), 1992, pp. 445-456.

[21] S. Hui and S. H. Zak, On discrete-time variable structure sliding mode control, Systems and Control Letters, vol. 38, 1999, pp. 283-288.

[22] G. Monsees, Discrete-time sliding mode control, Ph.D. Thesis, Delft University of Technology, The Netherlands, 2002.

[23] S. Boyd, L. E. Ghaoui, E. Feron, and V. Balakrishnan, Linear Matrix Inequalities in System and Control Theory, Society for Industrial and Applied Mathematics, Philadelphia, 1994.

[24] I. R. Peterson, A stabilization algorithm for a class of uncertain linear systems, Systems Control Lett. vol. 8 pp. 351357, 1987.

[25] N. Sharav-Scapiro, Z.J. Palmor, and A. Steinberg, Output stabilizing robust control for discrete uncertain systems, Automatica, vol. 34, no. 6, pp. 731-739, 1998

[26] Y. Niu and D.W.C. Ho, Design of sliding mode control subject to packet losses, IEEE Transactions on Automatic Control, vol. 55, no. 11, pp. 2623-2628, 2010.

[27] A. Bartoszewicz, Discrete-time quasi-sliding-mode control strategies, IEEE Transactions on Industrial Electronics, vol. 45, no. 4, pp. 633 637, 1998.

[28] S. Govindaswamy, S. K. Spurgeon and T. Floquet, Discrete-time output feedback sliding-mode control design for uncertain systems using linear matrix inequalities, International Journal of Control, vol. 84, no. 5, pp. 916930, 2011.

[29] H. K. Khalil, Nonlinear Systems, 3rd Edition, Prentice Hall, New York, 2002.

[30] W. Su, S.V. Drakunov and Ü. Özgüner, An $O\left(T^{2}\right)$ boundary layer in sliding mode for sampled-data systems, IEEE Transactions on Automatic Control, vol. 45(3), pp. 482-485, 2000. 